111 HEART FAILURE - THE EXPERIENCE OF LIVING WITH END-STAGE HEART FAILURE AND ACCESSING CARE ACROSS SETTINGS

Rosemary Chester, Heather Richardson, Christopher Doyle, Fiona Hodson, Joy R Ross. St Christopher's Hospice

10.1136/spcare-2021-PCC.129

Aims Heart failure is a complex clinical syndrome affecting an increasing number of the ageing population. Patients and carers require increasing input from specialist palliative care services to both manage symptoms and access support in the last year of life. An integrated clinical service between the local cardiology team at Princess Royal University Hospital and the palliative care team at St. Christopher's Hospice was piloted for patients with end-stage heart failure in Bromley in Kent, UK. This study explored views of patients and carers who participated in the integrated pilot service.

Methods and Results A qualitative study was conducted in which a convenience sample of patients and carers were invited to participate in focus groups: two bereaved carer groups $(n=2, n=2)$; one patient group $(n=4)$, held between 14th December 2018 and 18th January 2019. Participants were asked to describe their experiences of care received facilitated by a topic guide. Interviews were recorded, transcribed and coded using thematic analysis to identify common themes.

Four patients $(2: 2 \mathrm{M}: \mathrm{F})$ aged between 70 to 87 years and four female carers whom had cared for patients aged between 70 to 96 years who were since deceased, participated in this study. Overall, the service was positively received, and responses were mapped into four key areas; being diagnosed and living with heart failure, referral to palliative care, key helpful components of the care received and finally, unhelpful components of the new service in terms of care. Common themes emerged including understanding of heart failure and its trajectory, communication around palliative care, having a 'broker' for the system, recognition of carer's needs, service responsiveness, and feeling 'in control'.

Conclusions This qualitative study highlighted important considerations when developing an integrated heart failure and palliative care service. Education about heart failure for patients and carers, but also the integrated multidisciplinary team is crucial to improving detection of deterioration and facilitating communication around Advance Care Planning. The value of the 'expert-carer' should also be promoted and supported in chronic conditions. We recommend a focus on development of integrated services that enable joined-up care or single point of contact for patients and carers.

\section{SPECIALIST PALLIATIVE AND END OF LIFE TIMELY INTERVENTION PROJECT (SPELTIP) ON THE EMERGENCY FLOOR - OUTCOMES FROM THE FIRST 20 WEEKS}

Sarah Edwards, Lisa Keillor, Karen Murray, Stacey Kerslake, Julia Grant. University Hospitals of Leicester, Leicester Royal Infirmary; Kingsmill Hospital, Sherwood Forest Hospitals NHS Trust, Nottinghamshire

10.1136/spcare-2021-PCC.130
Background The majority of deaths occur in hospital. Most hospitals have access to Specialist Palliative Care with a variable amount of provision. Most are designed to be reactive and responsive.

A local audit in 2018 demonstrated that $89 \%$ of hospital deaths occurred following emergency admission. Clinical decision-making at admission did not always reflect the fact that the patient was at risk of dying. Opportunities to plan and involve patients in escalation decisions were missed and individualised planning was inconsistent. The aim was to improve the experience and outcomes for patients admitted with palliative and end of life care needs through role modelling, clinical support and education.

Methods A successful bid to Macmillan Cancer Support funded 2-year project for a proactive model of care with two Palliative Clinical Nurse Specialists (PCNS) based on the Emergency Floor. The Emergency Floor includes the Emergency Department and a variety of Acute Medical and Frailty wards. We wanted to evaluate the effectiveness of the PCNS service on staff and patients.

Results We report the first 20 weeks of the project, November 2019 to March 2020. The team had 336 contacts with 202 patients. $85 \%$ achieved their preference for place of care. The average length of stay was 3.75 days as opposed to 14 days for similar patients. Over $50 \%$ of patients did not need admission into the hospital from the Emergency Floor. Feedback has been extremely positive.

Conclusion Proactive specialist palliative care located at the point of admission improves the experience and outcomes for patients admitted with palliative and end of life care needs in an emergency. This work should influence how specialist services are provided in the future at a local and national level.

\section{IMPLEMENTING VIDEO CONSULTATIONS IN A UK HOSPICE DURING THE COVID-19 PANDEMIC: LEARNING FROM HEALTHCARE PROFESSIONALS' FEEDBACK}

Shannon Milne, Jennifer Palfrey, Jane Berg, Jennifer Todd. Princess Alice Hospice, Esher

\subsection{6/spcare-2021-PCC.131}

Introduction Providing care for our patients, carers and families during the coronavirus pandemic required a rapid shift to remote consultations, using videoconferencing technology. A service evaluation was performed to capture hospice professionals' experience.

Methods An online survey exploring experience and satisfaction was sent to a convenience sample of hospice professionals who had participated in video consultations between March and June 2020.

Results 35 hospice professionals responded, and both quantitative and qualitative data was analysed. $62.5 \%$ rated their video consultations as good, despite $94.1 \%$ having no prior video consultation experience. A third of respondents had undertaken potentially sensitive consultations, including advance care planning and resuscitation. Although 50\% of respondents had undertaken first assessments remotely, they consistently found this more challenging when the patient was not known to them previously. The results helpfully captured specific scenarios, when video consultations were less appropriate. $75 \%$ of respondents had undertaken a video 